\title{
Diseño instruccional desarrollado con estudiantes de pregrado en Matemáticas con el tema Expresiones numéricas
}

\author{
Claudia Lisete Oliveira Groenwald \\ claudiag@ulbra.br \\ http://orcid.org/0000-0001.7345-8205 \\ Universidade Luterana do Brasil (ULBRA) \\ Canoas, Rio Grande do Sul, Brasil.
}

Recibido: 26/04/2020 Aceptado: 18/05/2020

\begin{abstract}
Resumen
En la capacitación inicial, es importante que los estudiantes tengan la oportunidad de desarrollar las habilidades que les permitan actuar como profesionales capaces de desarrollar el proceso de enseñanza y aprendizaje. El objetivo de este artículo es presentar los resultados de un estudio de caso con estudiantes de Grado en Matemáticas cuando desarrollaron un Diseño Instruccional (DI) y cómo esto permite el desarrollo de las habilidades requeridas por el profesor profesional. El concepto de DI se aplicó en una situación didáctica con cinco estudiantes de pregrado en Matemáticas con el tema Expresiones numéricas, adecuado para estudiantes en el sexto año de la escuela primaria. El enfoque metodológico utilizado fue cualitativo con un enfoque en el estudio de caso. Los resultados muestran que fue posible observar que los estudiantes tuvieron la oportunidad de trabajar en las siguientes habilidades: trabajo grupal basado en la discusión, reflexión y acción para el desarrollo del DI; Evaluación y uso de tecnologías digitales, como materiales de estudio en PowerPoint y guardados en Ispring, software Jclic, actividades en línea; Comprender la importancia de la necesidad de conocer en profundidad los conceptos matemáticos para una planificación adecuada a la temática y al nivel de los estudiantes a los que se destina; valoración de metodologías de enseñanza para la construcción de conceptos matemáticos y el uso de recursos digitales; ser capaz de elegir tareas que sean apropiadas para los conceptos y la edad de los estudiantes para quienes el DI estaba destinado; Reflexión sobre la importancia de la evaluación constante durante todo el proceso y de la reorganización de las rutas cuando sea necesario.
\end{abstract}

Palabras clave: Educación Matemática. Formación inicial en Matemáticas. Diseño instruccional. Expresiones Numéricas.

\section{Design Instrucional desenvolvido com alunos de licenciatura em Matemática com a temática Expressões Numéricas}

\begin{abstract}
Resumo
$\mathrm{Na}$ formação inicial é importante que se possibilite que os estudantes tenham oportunidade de desenvolver as competências que os permitam atuar como profissionais capacitados ao desenvolvimento do processo de ensino e aprendizagem. O objetivo deste artigo é apresentar os resultados de um estudo de caso com estudantes de Licenciatura em Matemática ao desenvolveram um Design Instrucional (DI) e como isto possibilita o desenvolvimento das competências exigidas ao profissional professor. O conceito de DI foi aplicado em uma situação didática com cinco estudantes de Licenciatura em Matemática com o tema Expressões Numéricas, indicado para estudantes do $6^{\circ}$ ano do Ensino Fundamental. A abordagem
\end{abstract}


metodológica utilizada foi qualitativa com enfoque de estudo de caso. Os resultados apontam que foi possível observar que os estudantes tiveram oportunidade de trabalhar as seguintes competências: trabalho em grupo baseado na discussão, reflexão e ação para o desenvolvimento do DI; Avaliação e utilização das tecnologias digitais, como materiais de estudos no PowerPoint e salvo em Ispring, software Jclic, seleção de atividades online; compreensão da importância da necessidade de conhecer em profundidade os conceitos matemáticos para um planejamento adequado a temática e ao nível dos estudantes a que se destina; valorização das metodologias de ensino para a construção dos conceitos matemáticos e do uso de recursos digitais; valorizar a escolha de tarefas que sejam adequadas aos conceitos e a idade dos estudantes a que se destinava o DI; reflexão sobre a importância de avaliação constante durante todo o processo e de reorganização de rotas sempre que necessário.

Palavras-chave: Educação Matemática. Formação inicial em Matemática. Design Instrucional. Expressões Numéricas.

\title{
Instructional design developed with undergraduate students in Mathematics with the theme Numerical Expressions
}

\begin{abstract}
In initial training, it is important that students be given the opportunity to develop the skills that enable them to act as professionals capable of developing the teaching and learning process. The purpose of this article is to present the results of a case study with Mathematics Degree students when they developed an Instructional Design (DI) and how this enables the development of the skills required of the professional teacher. The DI concept was applied in a didactic situation with five undergraduate students in Mathematics with the theme Numerical Expressions, suitable for students in the 6th year of elementary school. The methodological approach used was qualitative with a focus on the case study. The results show that it was possible to observe that students had the opportunity to work on the following skills: group work based on discussion, reflection and action for the development of the DI; Evaluation and use of digital technologies, such as study materials in PowerPoint and saved in Ispring, Jclic software, online activities; understanding the importance of the need to know in depth the mathematical concepts for an adequate planning to the thematic and at the level of the students for which it is intended; valuation of teaching methodologies for the construction of mathematical concepts and the use of digital resources; become able to choose tasks that are appropriate to the concepts and age of the students for whom the DI was intended; reflection on the importance of constant evaluation throughout the process and of reorganizing routes whenever necessary.
\end{abstract}

Keywords: Mathematical Education. Initial training in Mathematics. Instructional Design. Numerical Expressions.

\section{Introdução}

Os cursos de licenciatura em Matemática, no Brasil, têm por objetivo formar os futuros professores em todas as suas potencialidades, com as competências exigidas para atuar na Educação Básica e atentos às demandas atuais da sociedade. A Resolução CNE/CP n 02 de 01 de julho (Brasil, 2015) focaliza à docência como ação educativa e como processo pedagógico intencional, envolvendo habilidades específicas, interdisciplinares além de conceitos, princípios 
e objetivos da formação que se desenvolvem na construção e apropriação dos valores éticos, linguísticos, estéticos e políticos do conhecimento inerentes à sólida formação científica e cultural do ensinar/aprender (Brasil, 2015).

Refletir sobre a formação de professores de Matemática implica discutir as características que definem o docente como profissional interessado e capacitado à criação e adaptação de métodos pedagógicos ao seu ambiente de trabalho, utilizando os conhecimentos matemáticos para a compreensão do mundo que o cerca e despertando no estudante o hábito pelo estudo independente, a criatividade, a persistência em resolver problemas, o interesse em conhecer seu ambiente de trabalho e seus estudantes, bem como, o hábito de refletir sobre seu trabalho docente, buscando caminhos que levem à uma educação de qualidade.

Neste sentido na formação inicial é importante que os professores desenvolvam experiências que possibilitem que os egressos sejam profissionais capacitados e com as competências que são indispensáveis ao desenvolvimento do processo de ensino e aprendizagem, considerando todos os aspectos referidos.

O objetivo deste artigo é apresentar os resultados de um estudo de caso, com estudantes de Licenciatura em Matemática ao desenvolveram um Design Instrucional (DI) e como isto possibilita o desenvolvimento de tais competências. O conceito de DI foi aplicado em uma situação didática com o tema Expressões Numéricas, indicado para estudantes do $6^{\circ}$ ano do Ensino Fundamental.

\section{Formação inicial}

Para Groenwald e Kaiber (2002) refletir sobre a formação de professores de Matemática implica discutir as características que definem o docente como profissional interessado e capacitado à criação e adaptação de métodos pedagógicos ao seu ambiente de trabalho, utilizando os conhecimentos matemáticos para compreensão do mundo que o cerca e despertando no aluno o hábito do estudo independente e a criatividade.

Segundo as Diretrizes Curriculares para os Cursos de Licenciatura em Matemática (Brasil, 2001) os egressos de um curso de Licenciatura devem ter, além de uma sólida formação de conteúdos matemáticos, uma formação pedagógica dirigida a sua prática que possibilite tanto a vivência crítica da realidade como a experimentação de novas propostas que considerem a evolução dos estudos da Educação Matemática e uma formação geral complementar envolvendo outros campos do conhecimento, necessários ao exercício da profissão. 
Nesse sentido as Diretrizes Curriculares indicam que os profissionais formados nos cursos de Matemática devem possuir uma visão abrangente do papel social do educador, abertura para aquisição e utilização de novas ideias e tecnologias, visão história e crítica da Matemática, capacidade de aprendizagem continuada e de trabalhar em equipes multidisciplinares, capacidade de comunicar-se matematicamente e compreender Matemática, de estabelecer relações com outras áreas do conhecimento, de utilizar os conhecimentos para compreensão do mundo que o cerca, capacidade de criação e adaptação de métodos pedagógicos ao seu ambiente de trabalho, de expressar-se com clareza, precisão e objetividade Deve, também, ser capaz de despertar o hábito da leitura e do estudo independente e incentivar a criatividade dos seus alunos.

A Resolução CNE/CP nº 02 de 01 de julho de 2015 (Brasil, 2015), que é a mais recente no que tange à formação nas licenciaturas, destaca que o aluno concluinte da graduação deve dominar o "[...] desenvolvimento, execução, acompanhamento e avaliação de projetos educacionais, incluindo o uso de tecnologias educacionais e diferentes recursos e estratégias didático-pedagógicas” (Brasil, 2015, p. 7). Além de “[...] relacionar a linguagem dos meios de comunicação à educação, nos processos didático-pedagógicos, demonstrando domínio das tecnologias de informação e comunicação para o desenvolvimento da aprendizagem" (Brasil, 2015, p. 8).

As competências específicas para um professor se referem a três dimensões fundamentais, as quais, de modo interdependente e sem hierarquia, se integram e se complementam na ação docente: conhecimento profissional; prática profissional; engajamento profissional. Apresentam-se no Quadro 1 as competências específicas de cada dimensão para a formação de professores. 
Quadro 1 - Competências das dimensões profissionais

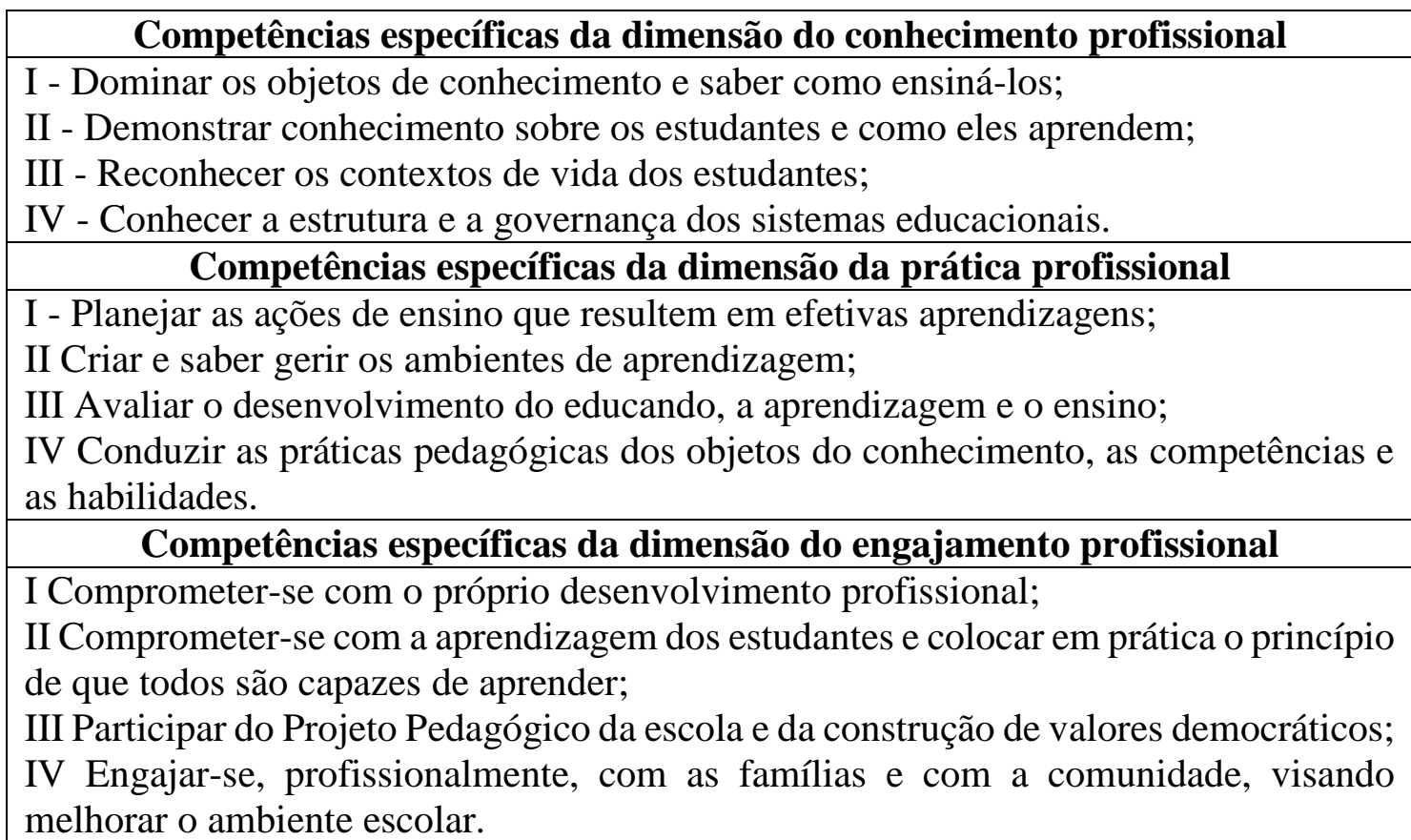

Fonte: Resolução CNE/CP nº 02 de 01 de julho de 2015 (BRASIL, 2015).

Segundo a Base Nacional Comum Curricular (BNCC) (Brasil, 2018), os estudantes devem desenvolver ao longo da Educação Básica dez competências, das quais frisa-se duas, que buscou-se salientar no estudo de caso apresentado neste artigo:

1. Utilizar diferentes linguagens - verbal (oral ou visual-motora, como Libras, e escrita), corporal, visual, sonora e digital -, bem como conhecimentos das linguagens artística, matemática e científica, para se expressar e partilhar informações, experiências, ideias e sentimentos em diferentes contextos e produzir sentidos que levem ao entendimento mútuo.

2. Compreender, utilizar e criar tecnologias digitais de informação e comunicação de forma crítica, significativa, reflexiva e ética nas diversas práticas sociais (incluindo as escolares) para se comunicar, acessar e disseminar informações, produzir conhecimentos, resolver problemas e exercer protagonismo e autoria na vida pessoal e coletiva.

Para que os professores em formação inicial tenham condições de integrar ao seu fazer pedagógico metodologias que utilizam tecnologias digitais é importante que tenham experiências positivas que os levem a reflexão e discussão destes pressupostos durante sua formação. 
Concorda-se com Kenski (2012) quando afirma que é importante discutir temas como conectividade, uso das mídias e redes sociais, enfim, a integração das Tecnologias Digitais (TD) na formação de professores.

Segundo Caccuri (2016) é importante que os professores sejam competentes para ensinar e aprender em um contexto de cultura digital. Entendendo cultura digital como um conjunto de saberes, crenças, usos e costumes que surgem na sociedade a partir da convergência das TIC, gerando uma revolução nos modos de acumular, apropriar-se e transmitir os dados, impulsionando novos desenvolvimentos sociais, políticos, econômicos e educativos.

As TD influenciam o currículo escolar, mudando a maneira de ensinar e influenciando no que se deve ensinar (NCTM, 2014). Porém, o uso eficaz das tecnologias requer conhecimento e um planejamento cuidadoso, exigindo dos docentes um desenvolvimento profissional adequado para poder utilizá-las em todo o seu potencial. Importante frisar que se o professor não conhece ou não está convencido da importância do uso destes recursos no desenvolvimento do processo de ensino e aprendizagem pode sentir-se inseguro a respeito do seu uso, não os incorporando ao currículo de um modo significativo.

Ressalta-se que se corrobora com as ideias de Bittar (2010) quanto a distinguir os termos inserção e integração das TD na Educação. Entende-se que professor insere as TD quando ela não provoca diferenças na aprendizagem, ou seja, a tecnologia é como se fosse um elemento estranho do fazer pedagógico. Entretanto, "integrar um novo instrumento (tecnologia digital) em sala de aula, implica mudanças pedagógicas, mudanças do ponto de vista da visão de ensino, que deve ser estudada e considerada pelos professores" (Bittar, 2010, p. 220).

Almeida e Valente (2011) salientam que a integração requer a reconstrução de conhecimentos por parte do estudante e, para tanto é necessário que o professor vivencie o processo de apropriação pedagógica das TD. Sob esse enfoque, o uso dos recursos das TD no processo de ensino e aprendizagem não ocorre apenas inserindo-os na sala de aula, mas integrando-os ao currículo, de modo a propiciar uma nova forma de ensinar (Bittar, 2010).

Levando estes pressupostos em consideração, no estudo de caso realizado com cinco estudantes em formação inicial, optou-se pelo planejamento de um Design Instrucional (DI) de Filatro $(2008 ; 2004)$ que se apresenta a seguir. 


\section{Design Instrucional}

Com a incorporação das Tecnologias Digitais (TD) na educação faz-se necessária uma ação sistemática do planejamento didático integrando o conteúdo, abordagem metodológica e recursos digitais, que sejam adequados ao desenvolvimento do processo de ensino e aprendizagem dos conteúdos e que permitam o desenvolvimento de competências.

Torna-se importante que os estudantes de Licenciatura, neste caso de Licenciatura em Matemática, sejam qualificados para um planejamento de acordo com as necessidades dos estudantes do século XXI.

Optou-se pelo desenvolvimento de um Design Instrucional (DI) de acordo com Filatro (2008) com o tema Expressões Numéricas indicado para estudantes do $6^{\circ}$ ano do Ensino Fundamental.

Segundo Filatro (2008) o DI é compreendido como o planejamento do ensino e aprendizagem, incluindo atividades, estratégias, sistemas de avaliação, métodos e materiais instrucionais. O DI é visto como um tipo de construção que envolve complexidade e síntese, podendo ser compreendido como a ação de estabelecer objetivos futuros e de encontrar meios e recursos para cumpri-los. Assim como o design, a palavra instrucional necessita de uma atenção bem específica para que não seja apenas identificada como instrução ou treinamento. Segundo Filatro (2008): “instrução é uma atividade de ensino que se utiliza da comunicação para facilitar a compreensão da verdade."

Apoiado por tecnologias, o DI admite mecanismos de efetiva contextualização, caracterizados por: maior personalização aos estilos e ritmos individuais de aprendizagem; adaptação às características institucionais e regionais; atualização a partir de feedback constante; acesso a informações e experiências externas à organização de ensino; possibilidade de comunicação entre os agentes do processo (professores, alunos, equipe técnica e pedagógica, comunidade); e monitoramento automático da construção individual e coletiva de conhecimentos (Filatro, 2004).

Neste sentido, foi utilizado o termo Design Instrucional Contextualizado (DIC) para descrever a ação intencional de planejar, desenvolver e aplicar situações didáticas específicas que, valendo-se das potencialidades de um Ambiente Virtual de Aprendizagem (AVA), incorporem, tanto na fase de concepção como durante a implementação, mecanismos que favoreçam a contextualização e a flexibilização, sendo possível que os estudantes percorram 
caminhos diferenciados, de acordo com seu ritmo de aprendizagem e de acordo com suas preferências de aprendizagem.

Segundo Filatro (2008) os modelos de DIC, frequentemente estruturam o planejamento do processo de ensino e aprendizagem em estágios distintos:

1. Análise: envolve a identificação de necessidades de aprendizagem, a definição de objetivos instrucionais e o levantamento das restrições envolvidas;

2. Design e desenvolvimento: quando ocorre o planejamento da instrução e a elaboração dos materiais e produtos instrucionais;

3. Implementação: quando se dá a capacitação e ambientação de docentes e alunos à proposta de design instrucional e a realização do evento ou situação de ensino e aprendizagem propriamente ditos; e por fim

4. Avaliação: envolve o acompanhamento, a revisão e a manutenção do sistema proposto. Apresenta-se na Figura 1 os movimentos de contextualização na proposta de DIC desenvolvida com o tema de estudo. Primeiro foi investigado os conceitos que envolvem o tema, estes foram organizados em um grafo com a sequenciação desejada de estudo de acordo com os objetivos propostos. Em um segundo momento foram investigadas as propostas de DIC que se adequam aos objetivos educacionais, o planejamento das atividades e recursos possíveis, também, como colocá-los no AVA escolhido para o experimento. No terceiro momento foram organizadas as sequências didáticas para cada conceito do grafo desenvolvido. A sequência didática envolve as ações: seleção das atividades com o tema, seleção da forma de acompanhamento do desempenho dos estudantes e como realizar a avaliação interna do DIC, replanejando quando necessário. 
Figura 1 - Ações desenvolvidas no DIC

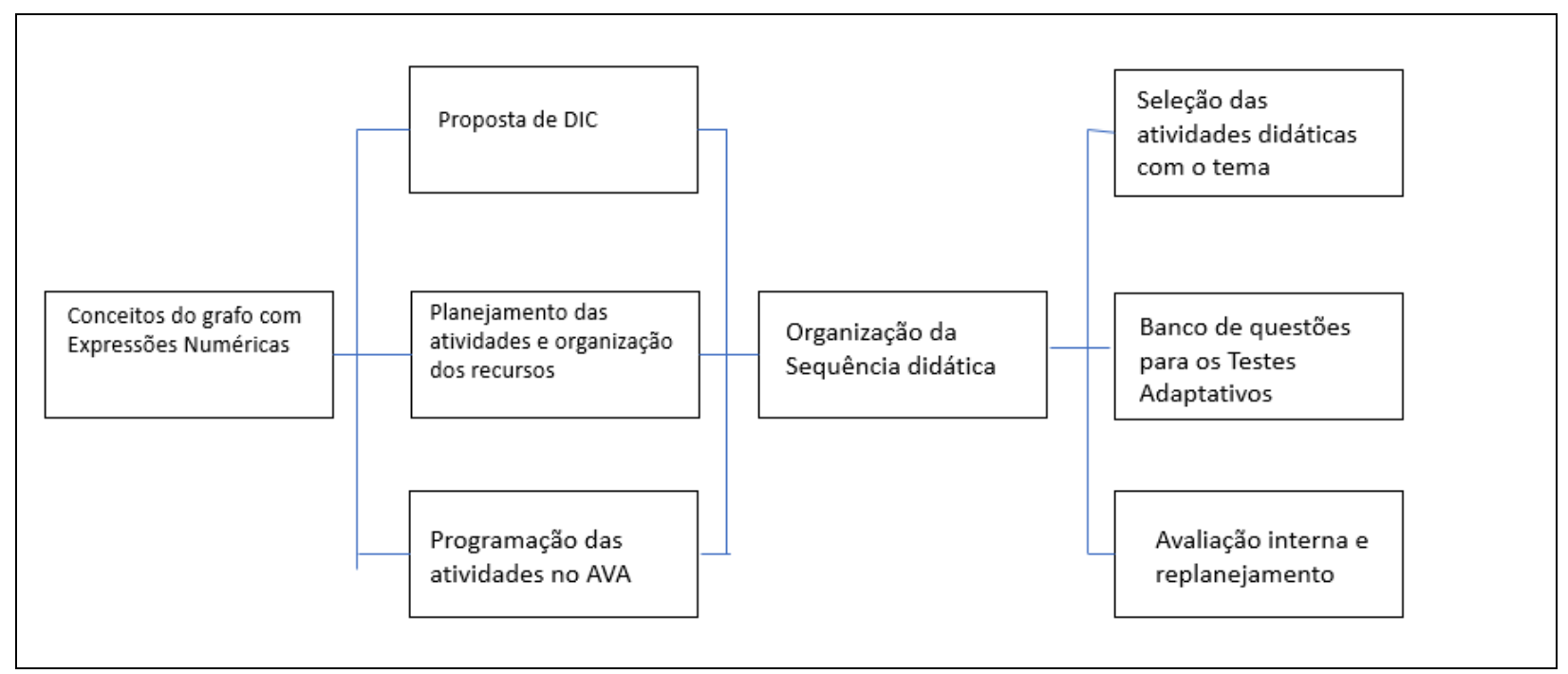

Fonte: Adaptado de Filatro (2004).

A seguir apresenta-se o percurso metodológico percorrido no estudo de caso e o DIC desenvolvido.

\section{Percurso metodológico}

Esta investigação foi desenvolvida em três etapas. A primeira, desenvolvida com reuniões mensais do Grupo de Estudos Curriculares de Educação Matemática (GECEM), cujos pesquisadores atuam no Programa de Pós-graduação em Ensino de Ciências e Matemática (PPGECIM) da Universidade Luterana do Brasil (ULBRA), buscando caminhos para a escolha de ações que possibilitassem uma abordagem metodológica com estudantes em formação inicial de Licenciatura em Matemática, que possibilitassem o desenvolvimento de competências que são indicadas para o desenvolvimento da profissão de professor e discussão das ações desenvolvidas. A segunda etapa foi a implementação (desenvolvimento, aplicação e avaliação) de um DIC com cinco estudantes de Licenciatura em Matemática da Universidade Luterana do Brasil, integrando Educação Matemática e Tecnologias Educacionais com o tema Expressões Numéricas. Na terceira e última etapa, após a conclusão do DIC, os registros foram retomados e analisados, em um processo de depuração das informações observadas e reconstrução da prática denominada reflexão-sobre-a-ação (Schön apud Almeida, 2000). Esse processo complementa a reflexão na ação, desenvolvida simultaneamente à ação instrucional na segunda etapa desta pesquisa. 
A abordagem metodológica utilizada foi qualitativa com enfoque no estudo de caso. Para Sampieri, Collado e Lucio (2013), a pesquisa qualitativa possibilita uma análise profunda dos dados, riqueza interpretativa, contextualização do ambiente e seu entorno, visto que se busca explicar as características e significados das informações obtidas através dos instrumentos de coleta de dados, como a observação não estruturada, as entrevistas e os registros escritos, mas sem a utilização de técnicas estatísticas para compreender as características ou comportamento estudados. Segundo Yin (2001, p. 33), entende-se “[...] estudo de caso como estratégia de pesquisa que compreende um método que abrange tudo com a lógica de planejamento incorporando abordagens específicas à coleta de dados e à análise de dados". Para Yin este tipo de investigação compreende o estudo aprofundado de um fenômeno no seu contexto real. Hernández, Fernández e Baptista (2006, p. 223 apud Ochoa, 2011, p. 72) afirmam que “[...] um estudo de caso constitui um método para aprender a respeito de uma instância complexa, baseado em um entendimento compreensivo desta instância como um 'todo' e seu contexto, mediante dados e informações obtidos por descrições e análises extensivas" (Tradução nossa).

Foram desenvolvidas as seguintes ações com os cinco estudantes de Licenciatura em Matemática:

1. Estudo da temática Expressões Numérica em livros didáticos do Ensino Fundamental e na Base Nacional Comum Curricular - BNCC (Brasil, 2018);

2. Análise e estudo do AVA, denominado SIENA, como ambiente para a programação do projeto;

3. Organização de um grafo com os conceitos a serem desenvolvidos no planejamento do DIC;

4. Construção de um banco de questões para compor os testes adaptativos de cada conceito do grafo desenvolvido para a avaliação dos estudantes durante o estudo dos conceitos do grafo;

5. Investigação e construção de atividades didáticas para compor as sequências didáticas de cada conceito do grafo desenvolvido e que permitissem o uso de recursos tecnológicos, com o delineamento de atividades com o tema Expressões Numéricas, proposto para o desenvolvimento de um DIC para estudantes do $6^{\circ}$ ano do Ensino Fundamental; 
6. Desenvolvimento do DIC, com o tema Expressões Numéricas para estudantes do $6^{\circ}$ ano do Ensino Fundamental;

7. Análise, discussão e replanejamento do DIC.

O objetivo do DIC foi de investigar uma abordagem didática, integrando o conteúdo matemático com uma abordagem didática com o uso de TD, visando o desenvolvimento de competências indicadas para o professor que vai atuar neste nível de ensino.

As atividades foram desenvolvidas em um ambiente utilizando tecnologias, utilizando um Ambiente Virtual de Aprendizagem (AVA), denominado SIENA (Sistema Integrado de Ensino e Aprendizagem).

As análises realizadas foram fundamentadas no DIC desenvolvido e nas opiniões dos cinco estudantes de Licenciatura em Matemática participantes da investigação. Os estudantes de Licenciatura em Matemática realizaram as atividades individualmente e em duplas. Os estudantes foram identificados como A1, A2, .., A5 e o pesquisador de P1.

O sistema SIENA foi desenvolvido pelos grupos de pesquisa: GECEM (Grupo de Estudos Curriculares de Educação Matemática), da Universidade Luterana do Brasil (ULBRA) e o Grupo de Tecnologias Educativas, da Universidade de La Laguna em Tenerife, Espanha. O SIENA é um sistema inteligente que permite que os estudantes realizem estudos individualizados e de acordo com suas dificuldades. Segundo Groenwald e Ruiz (2006), este sistema permite ao professor uma análise do nível de conhecimentos prévios de cada aluno, possibilitando um planejamento do processo do ensino e aprendizagem de acordo com a realidade dos alunos, podendo proporcionar uma aprendizagem significativa. O processo informático permite gerar um mapa individualizado das dificuldades dos alunos, o qual estará ligado a sequências didáticas, que servirão para recuperar as dificuldades que cada aluno apresenta no conteúdo desenvolvido.

As ações necessárias para o desenvolvimento de um experimento no SIENA são: grafo com os conceitos a serem desenvolvidos; banco de questões para os testes adaptativos para cada conceito do grafo; sequências didáticas eletrônicas para cada conceito do grafo.

\section{Experimento com alunos de Licenciatura em Matemática - DI com a temática Expressões} Numéricas

O experimento, com os 5 estudantes matriculados no $3^{\circ}$ semestre do curso de Licenciatura em Matemática, ocorreu em reuniões semanais de estudos e a reunião mensal com 
o grupo de pesquisa, durante os meses de março a dezembro de 2019, para discussão e organização das atividades para a construção do DIC.

O grafo com os conceitos a serem desenvolvidos foram organizados pelo pesquisador P1 e com todos os estudantes participantes do projeto. Os conceitos escolhidos como fundamentais para a compreensão do tema de estudo foram: Revisão do conceito e algoritmo de adição e subtração; Revisão do conceito e algoritmo de multiplicação e divisão; Revisão do conceito de Potenciação e Radiciação; Obstáculos Epistemológicos com Expressões Numéricas; Problemas envolvendo a necessidade de ordenar das operações; Problemas envolvendo a associação das operações; Problemas envolvendo Expressões Numéricas (Figura 3). No grafo o sistema SIENA lê as instruções de baixo para cima e de acordo com o nível de dificuldades indicado. O grafo não ordena os conceitos segundo relações arbitrárias, os conceitos são colocados de acordo com a ordem lógica em que devem ser apresentadas ao aluno. Portanto, o grafo deve ser desenvolvido segundo relações do tipo "o conceito A deve ser ensinado antes do conceito B”, começando pelos conceitos prévios, seguindo para os conceitos fundamentais, até atingir os conceitos objetivos.

Figura 2 - Grafo do Design de Expressões Numéricas

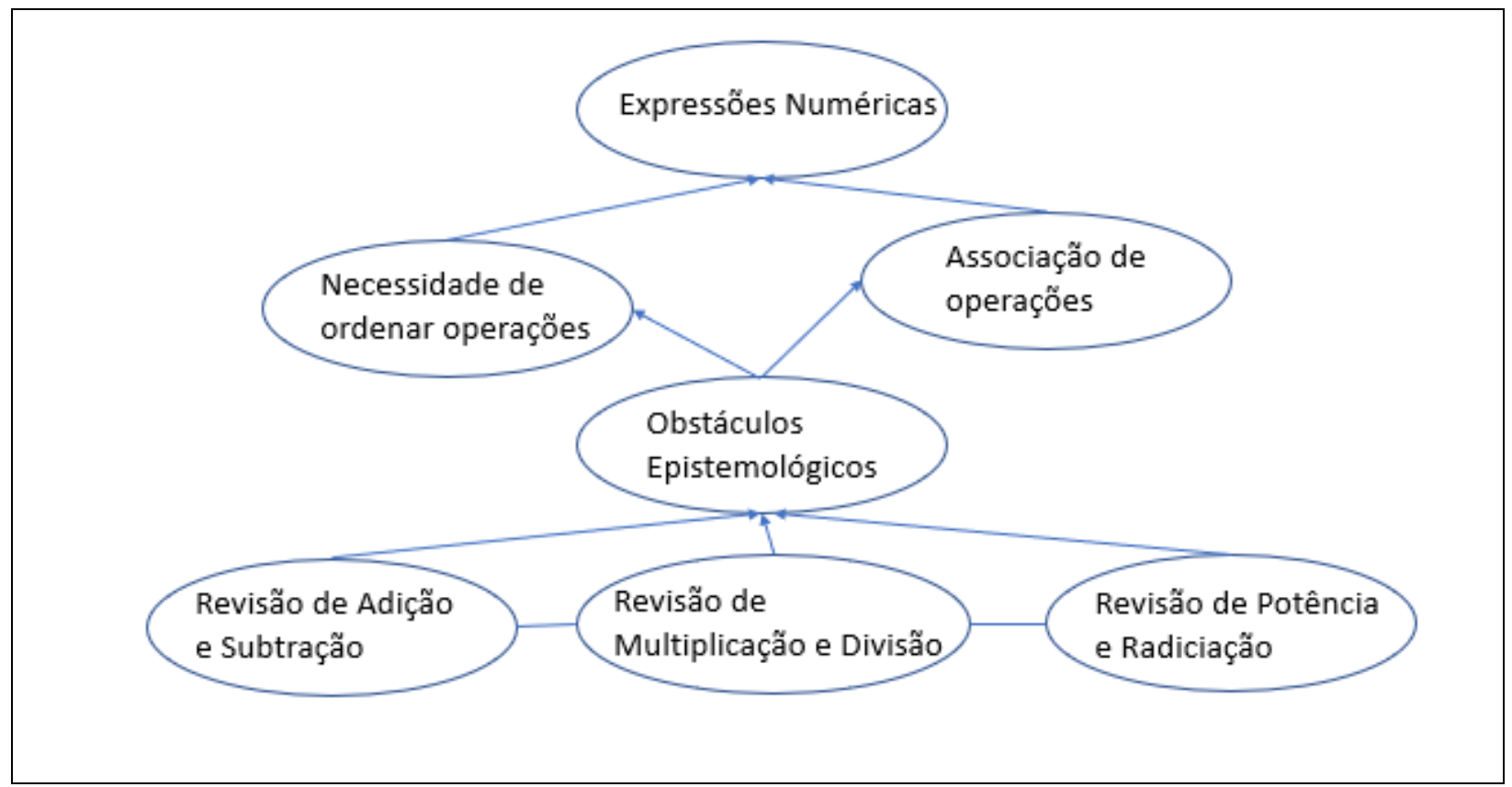

Fonte: http://siena.ulbra.br/expressoesnumericas. 
Os testes adaptativos informatizados são administrados pelo computador, que procura ajustar as questões do teste ao nível de habilidade de cada examinado. Segundo Costa (2009) um teste adaptativo informatizado procura encontrar um teste ótimo para cada estudante, para isso, a proficiência do indivíduo é estimada interativamente durante a administração dos testes e, assim, só são selecionados os itens que mensurem eficientemente a proficiência do examinado. $\mathrm{O}$ teste adaptativo tem por finalidade administrar questões de um banco de questões previamente calibradas, que correspondam ao nível de capacidade do examinado. Como cada questão apresentada a um indivíduo é adequada à sua habilidade, nenhuma questão do teste é irrelevante (Sands e Waters, 1997).

Para cada teste adaptativo foi construído um banco de questões com 45 questões, classificadas em níveis de dificuldade, sendo 15 questões consideradas de nível fácil, 15 questões de nível média e 15 questões de nível difícil, totalizando 315 questões. Apresenta-se a seguir exemplos do banco de questões investigadas, discutidas e avaliadas no estudo de caso desenvolvido (Quadro 2).

Quadro 2 - Exemplo de questões do banco de questões para os Testes Adaptativos com Expressões Numéricas ${ }^{1}$

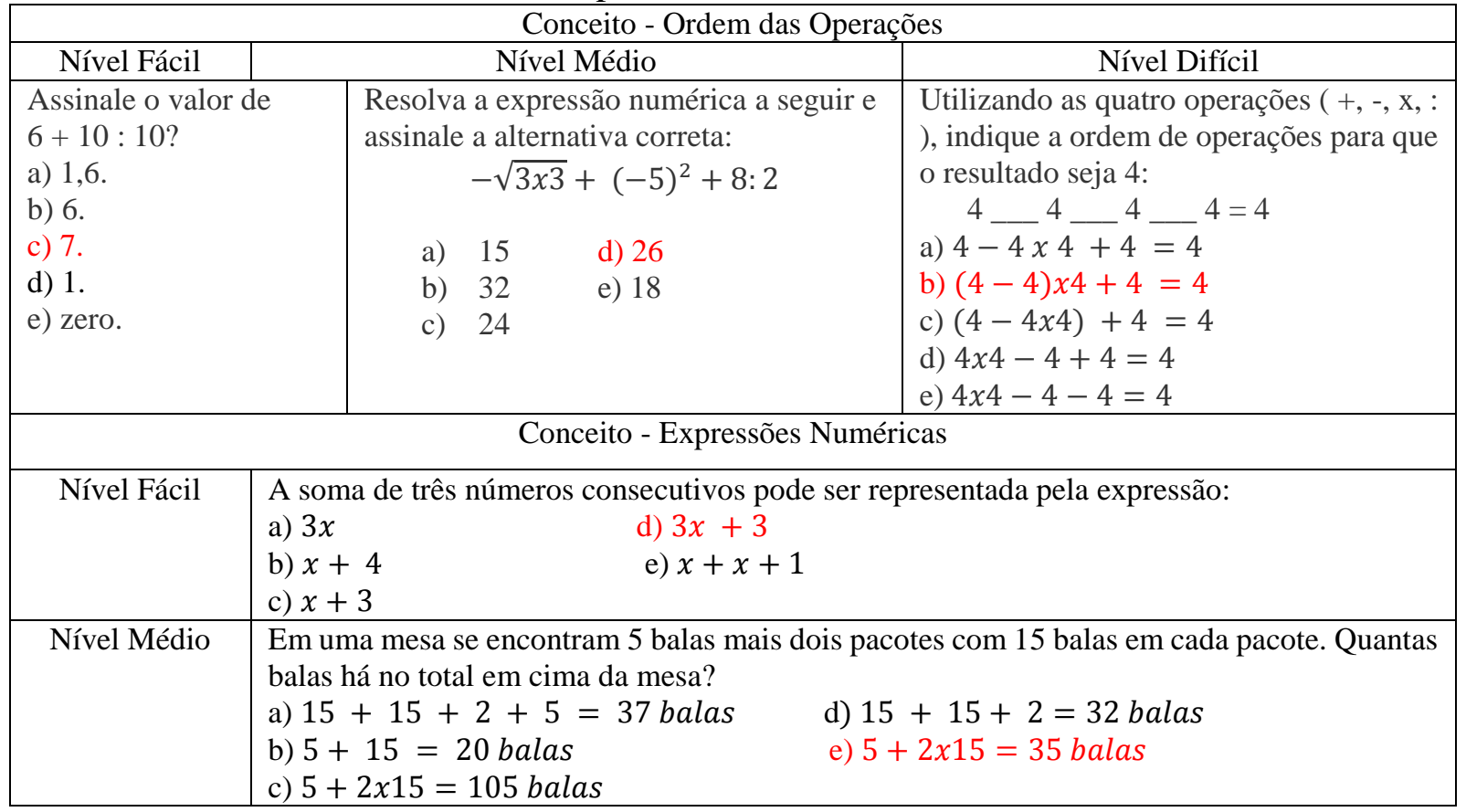

\footnotetext{
${ }^{1}$ As respostas em vermelho indicam a resposta correta.
} 
Quadro 2 - Exemplo de questões do banco de questões para os Testes Adaptativos com Expressões Numéricas ${ }^{2}$

continuação

\begin{tabular}{|c|l|}
\hline Nontinuação Difícil & $\begin{array}{l}\text { Os professores de uma escola precisavam fazer a contagem dos alunos vencedores dos jogos } \\
\text { internos a fim de adquirir as medalhas para premiação. No sexto ano, são } 60 \text { alunos no total. } \\
\text { Apenas a sexta parte deles recebeu medalhas no vôlei, e a metade recebeu medalhas no } \\
\text { futebol. No sétimo ano, com } 45 \text { alunos, apenas as meninas, que representam um terço dos } \\
\text { alunos da sala, foram premiadas no vôlei e todos os meninos foram premiados no futebol. Já } \\
\text { no oitavo ano, foram 7 medalhas de ouro, } 4 \text { de prata e } 3 \text { de bronze. Por fim, o nono ano não } \\
\text { participou da competição. Quantas medalhas foram compradas? }\end{array}$ \\
$\begin{array}{l}\text { a) } \frac{1}{6} \times 60+\frac{1}{2} x 60+\frac{1}{3} x 45+45+7+4+3=124 \text { medalhas } \\
\text { b) } 10+30+15+45+11=121 \text { medalhas } \\
\text { c) } \frac{1}{6} x 60+\frac{1}{2} x 60+\frac{1}{3} x 45+45+7+4+3=120 \text { medalhas } \\
\text { d) } \frac{1}{6} x 60+\frac{1}{2} x 60+\frac{1}{3} x 45+45=110 \text { medalhas } \\
\text { e) } \frac{1}{60} x 60+\frac{1}{2} x 60+\frac{1}{3} x 45+45+7+4+3=114 \text { medalhas }\end{array}$ \\
\hline
\end{tabular}

Fonte: http://siena.ulbra.br/expressoesnumericas.

As questões foram construídas e avaliadas pelos estudantes e pelo grupo de pesquisa, quando o banco de questões estava completo iniciou-se a construção das atividades didáticas para desenvolvimento das sequências didáticas. Todos os conceitos do grafo estão ligados a uma sequência didática que possibilita ao aluno estudar os conceitos ou realizar a recuperação dos conceitos em que apresenta dificuldades.

As sequências didáticas são um conjunto de atividades organizadas, de maneira sistemática, planejadas para o processo de ensino e aprendizagem de um conteúdo, etapa por etapa. São organizadas de acordo com os objetivos que o professor quer alcançar para a aprendizagem de seus alunos, e envolvem atividades de aprendizagem e avaliação (Dolz e Schneuwly, 2004). Segundo Zabala (1998) as sequências didáticas são um conjunto de atividades ordenadas, estruturadas e articuladas para a realização de certos objetivos educacionais, que têm um princípio e um fim conhecidos tanto pelos professores como pelos alunos. Através da sequência didática é possível analisar as diferentes formas de intervenção e avaliar a pertinência de cada uma delas.

Nas sequências foram desenvolvidos materiais com os seguintes recursos didáticos: material de estudos em PowerPoint salvo em Ispring; atividades didáticas no Jclic; atividades online com jogos com expressões. JClic é um software de autoria, na língua espanhola e catalã. Trata-se de uma ferramenta desenvolvida na plataforma Java5, para criação, realização e

\footnotetext{
${ }^{2}$ As respostas em vermelho indicam a resposta correta.
} 
avaliação de atividades educativas multimídias. É uma aplicação de software livre, baseada em modelos abertos em diversos ambientes operacionais (Linox, Mac OS-X, Windows e Solaris).

A seguir apresenta-se exemplos dos materiais de estudos desenvolvidos (Figura 3).

Figura 3 - Material de Estudos com Expressões Numéricas

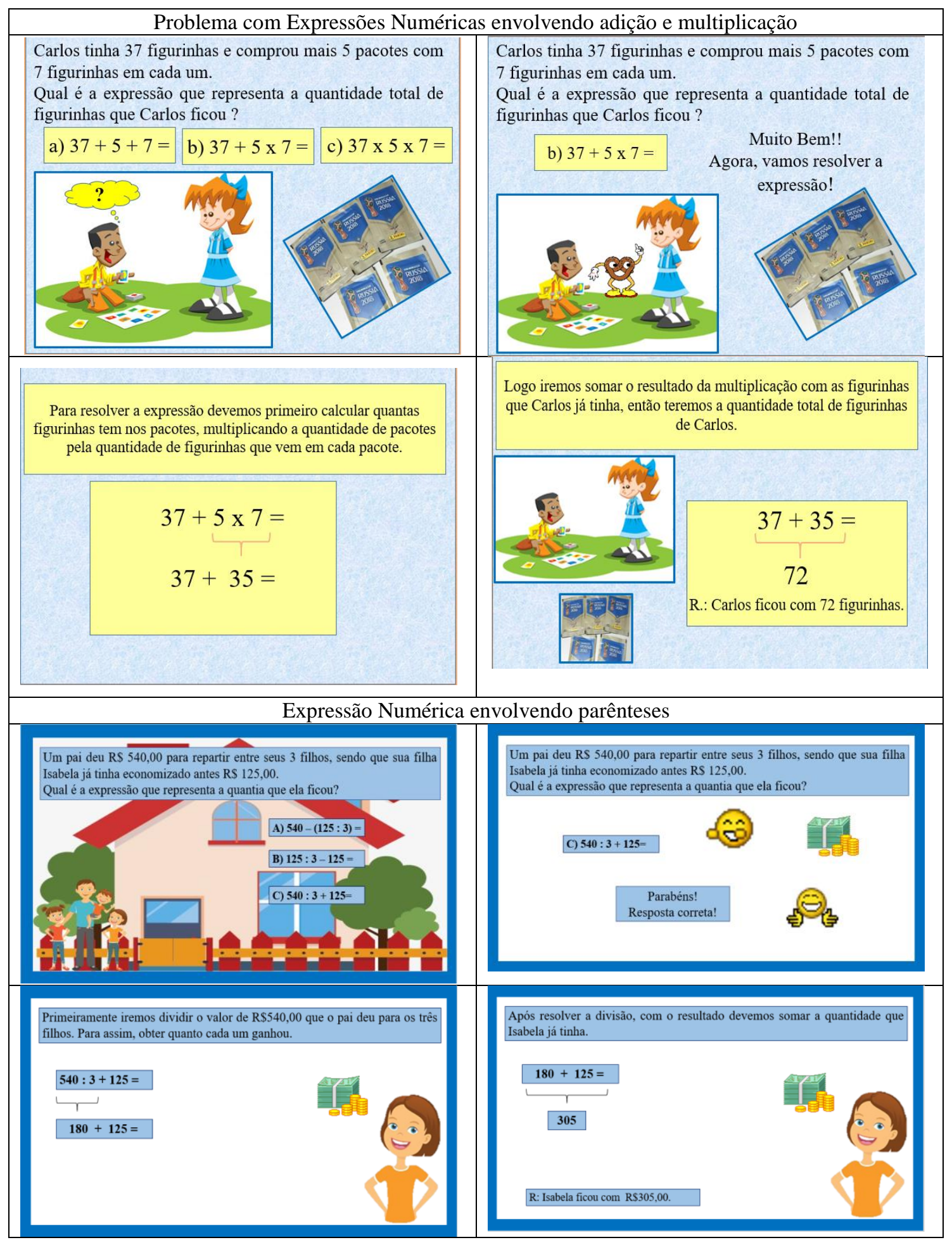


Figura 3 - Material de Estudos com Expressões Numéricas (continuação)

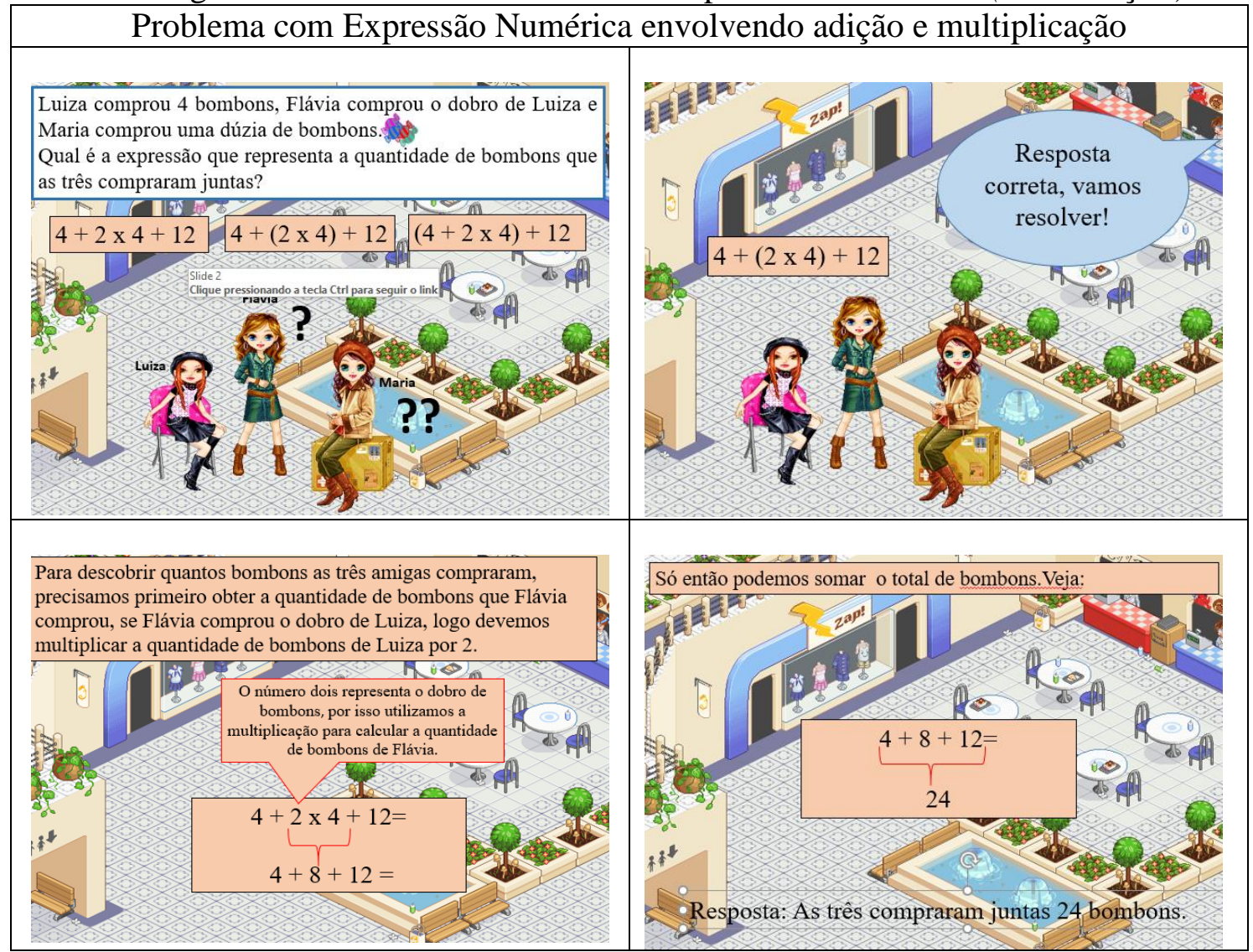

Fonte: http://siena.ulbra.br/expressoesnumericas.

Também foram desenvolvidas atividades com o software Jclic (Figura 4).

Figura 4 - Atividades didáticas no software Jclic

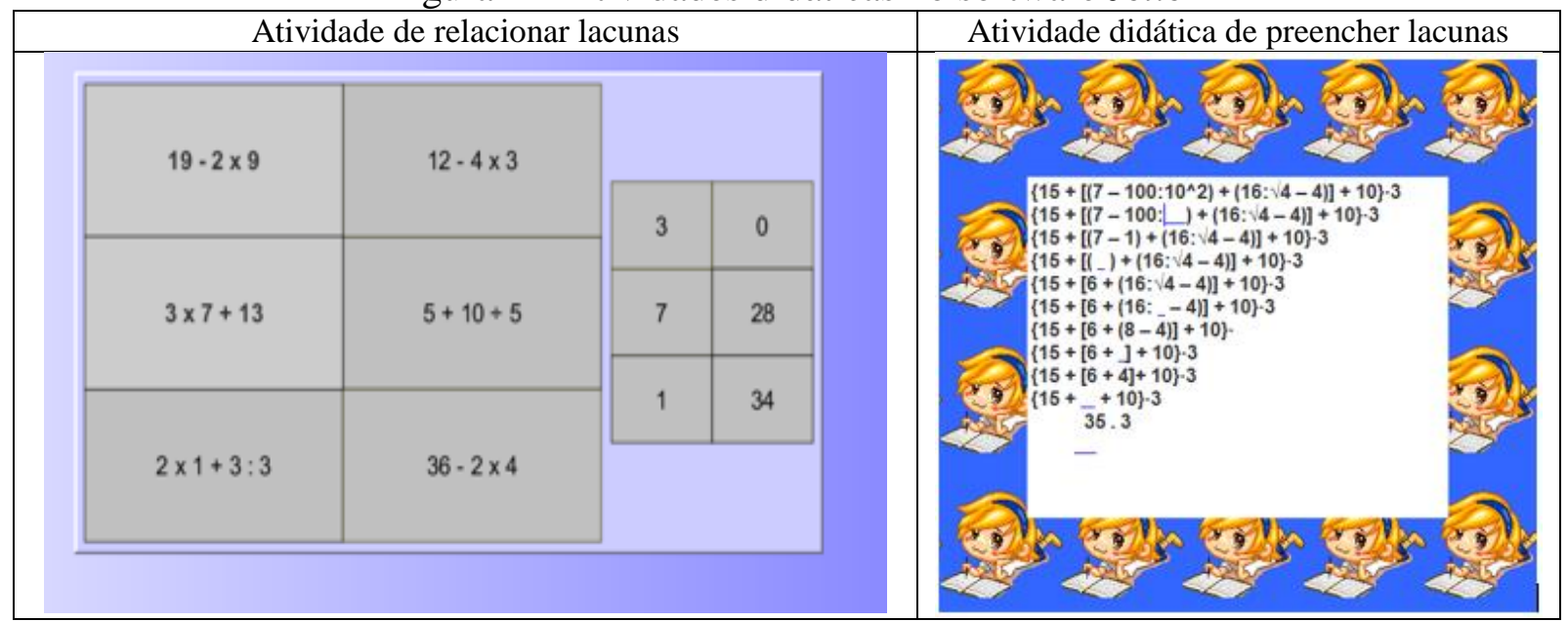

Fonte: http://siena.ulbra.br/expressoesnumericas 
A plataforma SIENA está disponível no endereço http://siena.ulbra.br, sendo que o acesso aos trabalhos e banco de dados está restrito a usuários cadastrados no sistema. Esse cadastramento é realizado pelos administradores da plataforma, e fornece um login e uma senha pessoal ao usuário.

\section{Análise dos dados}

O processo de construção do DIC seguiu as etapas de Filatro (2008; 2004) e os estudantes realizaram um processo de reflexão-ação.

As estudantes A1 e A2 ficaram responsáveis pelo estudo do software Jclic e para que repassassem estes para todos do grupo, bem como da construção do banco de questões dos três primeiros conceitos do grafo; As estudantes A3, A4 e A5 ficaram responsáveis pela investigação das atividades didáticas com Expressões Numéricas e apresentarem ao grupo para análise, discussão e reflexão da validade das mesmas e construção do banco de questões dos outros três banco de questões.

Observou-se que a troca de informações sobre as investigações realizadas e o processo de discutir, refletir e avaliar, pelos colegas, o processo desenvolvido permitiu um processo de reflexão-ação que foi positivo ao grupo no sentido de aceitar críticas, avaliar e replanejar o trabalho desenvolvido, possibilitando qualificar as atividades e reorganizar nos recursos digitais escolhidos.

Após vários momentos de reflexão e discussão as estudantes decidiram que os conceitos considerados obstáculos epistemológicos seriam: operações com os elementos neutros na adição e na multiplicação; potenciação envolvendo o zero, o número um e o expoente zero; radiciação envolvendo números negativos. A pesquisadora P1 identificou que estes conceitos, também, se constituíam obstáculos para as alunas participantes do experimento.

As alunas A2, A4 e A5 não apresentavam compreensão do uso dos parênteses em uma expressão numérica. Exemplo do uso indevido de parênteses: $(3 \times 4)+(4: 4)$. A alegação das estudantes é que estavam habituadas a utilizar parênteses em todas situações, evitando o erro.

A estudante A5 afirmou que as expressões $(7-7) x 7+7$ e $7-7 x 7+7$ apresentavam o mesmo resultado. $\mathrm{O}$ que levou a muitas discussões entre as estudantes e a pesquisadora para que reconhecessem o erro e encontrassem caminhos didáticos para que, futuramente, como professores realizassem planejamentos que identificassem tais dificuldades em seus alunos e pudessem saná-las com atividades adequadas. 
As alunas apresentaram dificuldades na elaboração do material de estudos quando deveriam desenvolver explicações que identificavam a ordem das operações sem apresentarem as regras e sim, por meio de problemas onde ficasse evidente ao estudante realizar as operações na ordem correta. Um grande desafio foi o replanejar e refazer as atividades, pois os recursos tecnológicos escolhidos exigiram esforço, investigação e readaptação das atividades em uma trajetória complexa e desafiadora. Até nas atividades simples como o exemplo da figura 5, foram muito discutidas para que as estudantes compreendessem o caminho didático a ser escolhido.

Figura 5 - Exemplo de dificuldades das estudantes no caminho metodológico a ser escolhido

\begin{tabular}{|l|l|l|}
\hline \multicolumn{3}{|c|}{ Problema com multiplicação e adição } \\
\hline $\begin{array}{l}\text { Sarah tem } 5 \text { laranjas e as colocou sobre a mesa. Sua mãe } \\
\text { trouxe } 3 \text { bacias com } 7 \text { laranjas cada uma. } \\
\text { Qual é a expressão que representa a quantidade de } \\
\text { laranjas que ficaram em cima da mesa? }\end{array}$ \\
\hline a) $5+3+7=0$ b) $5+3 \times 7=0$ \\
\hline $5+3 x 7=26$ laranjas $5+7=$ \\
\hline $\begin{array}{l}\text { As estudantes tiveram dificuldades em encontrar } \\
\text { argumentação para que os estudantes percebessem } \\
\text { que primeiro deveriam realizar a multiplicação e e } \\
\text { depois adicionar o resultado com as cinco laranjas. }\end{array}$
\end{tabular}

Fonte: Observações realizadas P1 durante o estudo de caso.

Todas as estudantes afirmaram que se sentiam mais confiantes depois do experimento que participaram desenvolvendo o DIC e que, com certeza, estavam mais preparadas para planejamentos didáticos com o uso de TD.

Cada fase do DIC, cada recurso tecnológico disponibilizado, cada situação didática relatada comporta numerosos desafios a serem desvendados e oportunidades renovadas de articulação entre teoria e prática pedagógica. 


\section{Considerações finais}

O trabalho desenvolvido consistiu na possibilidade de compreender como um planejamento pode integrar os conceitos educacionais aqui apresentados, associando-as às questões de uso das TD no contexto educacional e como o desenvolvimento de um DIC possibilita em estudantes de Licenciatura em Matemática o desenvolvimento de competências profissionais esperadas para um professor de Matemática.

Entende-se que foi possível o desenvolvimento de competências, não completamente, mas um processo de desenvolvimento, que deve ser contínuo e ao longo da formação inicial e que deve seguir durante a vida profissional destes estudantes. É possível salientar que as principais competências trabalhadas ao longo do processo foram: trabalho em grupo baseado na discussão, reflexão e ação para o desenvolvimento do DIC; Avaliação e utilização das TD como materiais de estudo no PowerPoint e salvo em Ispring, software Jclic, atividades online; compreensão da importância da necessidade de conhecer em profundidade os conceitos matemáticos para um planejamento adequado a temática e ao nível dos estudantes a que se destina; valorização das metodologias de ensino para a construção dos conceitos matemáticos e do uso de recursos digitais; escolha de tarefas que sejam adequadas aos conceitos e idade dos estudantes a que se destinava o DIC; reflexão sobre a importância de avaliação constante durante todo o processo e de reorganização de rotas sempre que necessário.

Outro ponto importante que foi perceptível durante o processo foi a conscientização de que um planejamento não é único e que exige mudanças de rumos. Muitas vezes foi necessário alterar as atividades escolhidas, os recursos escolhidos e a forma de organizá-los em uma sequência didática, sendo necessário avaliação interna constante. Um exemplo, que se pode citar, foi a organização dos problemas de expressões em vídeo e depois se entendeu importante a gravação de voz, com explicações sobre as possibilidades de resolução, o que levou a necessidade de realizar novamente os vídeos.

Importante salientar que o professor de Matemática pode agir para que a escola se transforme em um espaço rico de aprendizagem significativa, presencial e digital, que levem os alunos à proatividade, a aprender a aprender, tomar iniciativa e interagir segundo os princípios citados por Morán, Maseto e Beherens (2013). 


\section{Agradecimentos}

Ao CNPq e a FAPERGS pelas bolsas de Iniciação Científica que possibilitaram esta investigação no Programa de Pós-graduação em Ensino de Ciências e Matemática (PPGECIM) na Universidade Luterana do Brasil (ULBRA).

\section{Referências}

ALMEIDA, M. E. B. de (2000). Informática e formação de professores. In: Cadernos Informática para a mudança em educação. Brasília, Ministério da Educação/SEED.

ALMEIDA, M.E.B.; VALENTE, J.A. (2011). Tecnologias e currículo: trajetórias convergentes ou divergentes? São Paulo: Paulus.

BITTAR, M. A. (2010). Escolha de um software educacional e a proposta pedagógica do professor: estudo de alguns exemplos da matemática. In: W. Beline; N.M. Lobo Da Costa. Educação Matemática, tecnologia e formação de professores: algumas reflexões (p. 215242). Campo Mourão: Editora FECILCAM.

BRASIL, (2018). Ministério da Educação. Base Nacional Comum Curricular - Versão final. Brasília: MEC. Recuperado de:

BRASIL. MEC. (2001). Diretrizes Curriculares para Cursos de Matemática. MEC. Acesso em abril de 2019: http://portal.mec.gov.br/cne/arquivos/pdf/CES13022.pdf.

BRASIL. MEC. (2019). Resolução CNE/CP $N^{o}$ 2, de 20 de Dez de 2019. Acesso em: 10 de fevereiro de 2020.

CACCURI, V. (2016). Tecnologia Digital para docentes: Computación y TICs em el aula. Buenos Aires: Dalaga.

COSTA, Denise Reis. (2009). Métodos estatísticos em testes adaptativos informatizados. 2009. 107 f. Dissertação (Mestrado em Estatística) - Instituto de Matemática, Universidade Federal do Rio de Janeiro, Rio de Janeiro.

DOLZ, J.; SCHNEUWLY, B. (2004). Gêneros orais e escritos na escola. Campinas/SP: Mercado das Letras.

FILATRO, A. (2008). Design Instrucional na prática. São Paulo: Pearson.

FILATRO, A. e STELA, C. B. P. (2004). Design Instrucional Contextualizado. Revista da Faculdade de Educação da USP. Abril/2004. Acesso em fevereiro de 2020. http://www.miniweb.com.br/atualidade/Tecnologia/Artigos/design_instrucional.pdf.

GROENWALD, C. L. O.; KAIBER, C. T. (2002). Educação matemática na formação dos professores. Educação Matemática em Revista - RS, Rio Grande, n. 4, p. 64-6.

GROENWALD, C. L. O.; RUIZ, L. M. (2006). Formação de Professores de Matemática: uma proposta de ensino com novas tecnologias. Acta Scientiae, Canoas, v.8, n.2, jul./dez.

http://basenacionalcomum.mec.gov.br/images/BNCC_EI_EF_110518_versaofinal_site.pdf KENSKI, V. M. (2012). Tecnologias e Ensino Presencial e a Distância. São Paulo: Papirus. 
MORÁN, J; MASETTO, M. T.; BEHRENS, M. (2003). Novas Tecnologias e Mediação Pedagógica. Campinas: Papirus, $21^{\mathrm{a}}$ ed.

NCTM. (2014). Principles to actions: ensuring mathematical sucess for all. Reston: National Council of Teachers of Mathematics.

OCHOA, J. A. V. (2011). La comprensión de la tasa de variación para uma aproximación al concepto de derivada. Uma análise desde la teoria de Pirie y Kieren. 228 f. Tese (Doutorado em Educação) - Departamento de Educação Avançada, Medellín, Antioquia, 2011. Disponível em: < http://www.rc.unesp.br/gpimem/downloads/teses/lacompresiontasadevariacion.pdf >. Acesso em: 15 out. 2014.

SAMPIERI, R. H.; COLLADO, C. F.; LUCIO, M. del P. B. (2013). Metodologia de pesquisa. $5^{\text {a }}$ edição, Porto Alegre: Penso.

SANDS, W. A.; WATERS, B. K. (1997). Introduction to ASVAB and CAT. In: SANDS, William A.; WATERS, Brian K.; MCBRIDE, James R. (Eds.). Computerized adaptive testing: from inquiry to operation. Washington: American Psychological Association.

YIN, R. K. (2009). Case study research, Design and methods. Thounsand Oaks, California: Sage Publications, Inc.

ZABALA, A. (1998). A prática educativa: como ensinar. Porto Alegre: Artmed. 